\title{
Nutrición y rendimiento en atletas con discapacidad intelectual y conocimientos de sus cuidadores
}

\author{
Blanca Estela Hernández Martínez,* Ana Olivia Caballero Lambert,* Ana Karen Estrada Salas,* \\ Martha Leticia Guevara Sanginés**, Judith Orozco Méndez****
}

\begin{abstract}
RESUMEN
Introducción: Se ha demostrado que la población con discapacidad intelectual presenta factores de riesgo en cuanto a salud y nutrición; sin embargo, se cuenta con pocos estudios que caractericen esta problemática. Objetivo: Determinar el consumo alimentario, diagnóstico nutricio, rendimiento físico y estado de hidratación en 179 atletas con discapacidad intelectual afiliados a las Olimpiadas Especiales de Guanajuato, provenientes de nueve municipios, así como el nivel de conocimientos y hábitos de hidratación de los padres o cuidadores. Metodología: Estudio descriptivo, prospectivo y transversal. El consumo alimentario se obtuvo mediante un cuestionario semicuantitativo validado (el Sistema de evaluación de hábitos nutricionales y consumo de nutrimentos, SNUT); el diagnóstico nutricio se determinó mediante parámetros antropométricos; el rendimiento físico fue evaluado por las pruebas de DIDEFI ${ }^{\circledast}$; el estado de hidratación fue determinado por la densidad urinaria mediante refractómetro; y el nivel de conocimientos, hábitos de hidratación y nutrición fueron evaluados por dos cuestionarios validados. Resultados: Se reporta que el grupo de edad de 11 a 20 años presenta los valores más altos tanto en calorías como en macronutrimentos. Con relación al estado de nutrición, el $43.6 \%$ presenta algún grado de exceso de peso y el $4.5 \%$ bajo peso. Se encontró en las pruebas físicas una tendencia al crecimiento monotónico en la fuerza de la mano y abdominales por minuto, una tendencia parabólica en el salto horizontal y una representación azarosa en la flexión del tronco. El 56.42\% de los atletas presentaron deshidratación. Se observó que la media en conocimientos es mayor que la calificación del cuestionario de hábitos de hidratación, con diferencia estadísticamente significativa. Conclusiones: Los atletas con discapacidad intelectual deben considerarse como un grupo de riesgo por presentar factores como alteraciones en el peso y deshidratación; además la falta de conocimientos en nutrición e hidratación por parte de sus padres puede poner en riesgo su salud y desempeño deportivo.
\end{abstract}

Palabras clave: Diagnóstico nutricio, rendimiento físico, estado de hidratación, atletas con discapacidad intelectual.

\section{Nutrition and physical performance in athletes with intellectual disability and knowledge level of their caregivers}

\begin{abstract}
Introduction: It has been proven that the population with intellectual disability presents health and nutrition risk factors; however, there are only a few studies that characterize this problematic situation. Objective: The purpose of this study was to determine the food consumption, nutritional diagnosis, physical performance and hydration status of 179 athletes with intellectual disability affiliated to the Guanajuato Special Olympics, from nine cities, as well as the knowledge level and hydration habits of parents or caregivers. Methodology: A descriptive, prospective and transversal study has been con-

\footnotetext{
* Universidad Iberoamericana León.

** Universidad de Guanajuato.

*** Universidad Iberoamericana León.

Correspondencia: Blanca Estela Hernández Martínez. Universidad Iberoamericana León. Bulevar Jorge Vertiz Campero Núm. 1640, Col: Cañada de Alfaro. 37238, León, Gto. Tel: 71000 00, ext. 2050.

E-mail: blanca.hernandez@leon.uia.mx

Este artículo puede ser consultado en versión completa en http://www.medigraphic.com/enfermerianeurologica
} 
ducted. Food consumption was obtained through a validated semi-quantitative questionnaire; the nutrition diagnosis was determined by anthropometric parameters; physical performance was evaluated by the tests DIDEFI ${ }^{\circledR}$; the hydration status was calculated by urinary density using a refractometer; and the knowledge level, hydration and nutrition habits of the caregivers were assessed by two validated questionnaires. Results: The age group 11 to 20 years has the highest calorie and macronutrient ingestion. In relation to the nutritional status: $43.6 \%$ is overweight in some degree and $4.5 \%$ underweight. A tendency was found in the physical tests to monotonic growth in hand strength and abdominals per minute, a parabolic tendency in horizontal jump and a random representation in trunk flexion. $56.42 \%$ of the athletes presented dehydration. Regarding the parents/caregivers, their average knowledge was higher than their grade in the hydration habits questionnaire, with a significant statistics difference. Conclusions: The athletes with intellectual disabilities must be considered as a risk group because of factors such as alterations in weight and dehydration, as well as their parents'/caregivers' lack of knowledge in nutrition and hydration, which could risk their health and physical performance.

Key words: Nutrition diagnosis, physical performance, hydration status, athletes with intellectual disability.

\section{PLANTEAMIENTO DEL PROBLEMA}

i Cuál es el diagnóstico nutricio, rendimiento físico y estado de hidratación de atletas con discapacidad intelectual pertenecientes a las Olimpiadas Especiales de Guanajuato, de las delegaciones de León, Guanajuato, Celaya, Cortázar, Comonfort, Salamanca, Irapuato, San Miguel de Allende y Moroleón, además del nivel de conocimientos y prácticas alimentarios en sus padres y/o cuidadores, de enero a diciembre de 2012 ?

\section{INTRODUCCIÓN}

La Organización Mundial de la Salud señala que 'discapacidad' es un término general que abarca las deficiencias, limitaciones de la actividad y restricciones de la participación, y que a nivel mundial cerca del $10 \%$ de la población tiene alguna discapacidad. En México, en el año 2010, las personas con algún tipo de discapacidad eran el 5.1\% de la población total..$^{1-2}$

La discapacidad intelectual (DI) puede ser causada por una lesión, enfermedad o anormalidad cerebral. El síndrome de Down (SD) es la causa más frecuente de retraso. De la misma manera, una persona con parálisis cerebral, autismo, síndromes de Noonan, Williams Beuren o déficit de atención e hiperactividad (SDAH) puede presentar discapacidad intelectual. $^{3}$

Las personas con DI presentan factores de riesgo de salud y nutrición. Con relación a ellos, en el SD pueden manifestarse cardiopatía congénita, constipación, enfermedad celiaca e hipotiroidismo. Los niños con parálisis cerebral muestran una elevada prevalencia de desnutrición. Las personas con autismo tienen hábitos alimentarios diferentes a los de la población normal, incluyendo pica; pueden presentar deficiencia de fibra dietaria, calcio, hierro, vitamina E y D. En el síndrome de Williams Beuren aparecen hipercalcemia y alteraciones renales y vasculares. En cuanto al SDAH, como parte del tratamiento se utilizan psicoestimulantes que conllevan a la disminución del apetito. ${ }^{3}$

La relación entre la dieta y el rendimiento deportivo es importante porque cada nutrimento juega un papel específico en el desempeño físico y en la recuperación. ${ }^{4}$ En un estudio de Fernández, se encontró que el 100\% de los atletas presentaron un consumo de calorías mayor al porcentaje de adecuación del 90-110\%; en cuanto a los macronutrimentos, el $100 \%$ de los sujetos presentaron un porcentaje elevado de lípidos y disminución en hidratos de carbono y proteínas. ${ }^{5}$

La composición corporal en las personas con DI puede tener diferencias en relación con las personas sin este tipo de impedimento. ${ }^{3}$ En relación con los parámetros antropométricos, Fernández reportó que, con respecto al índice de masa corporal (IMC), el $100 \%$ de las participantes del sexo femenino con SD presentaron sobrepeso. ${ }^{5}$

En cuanto al rendimiento físico, Bofill evaluó la condición física de la población con SD, DI, sin SD y población general, donde encontró los mejores resultados para la población general. ${ }^{6}$

Aunado a un adecuado estado nutricio, el mantenimiento del estado de hidratación es importante para lograr un apropiado rendimiento físico. Algunos estudios reportan que aun en ejercicios de corta duración (20 a 30 minutos) existe pérdida importante de líquido, además de que existen malas prácticas de hidratación. La falta de conocimientos sobre el tema constituye un factor determinante. En un estudio realizado a deportistas infantiles y juveniles sin DI, se encontró que solo el 9\% contaba con apropiados conocimientos de hidratación, y que el $62.22 \%$ de la población no estaba en condiciones óptimas de hidratación. ${ }^{6,7}$

En lo relacionado con los cuidadores principales de personas con DI, existen pocos estudios sobre su conocimiento y prácticas alimentarias. Sánchez reportó que, en un estudio 
realizado a escolares sin DI y sus padres, un porcentaje alto no conocía la imagen del plato del bien comer. ${ }^{8}$

Una de las limitaciones para obtener un adecuado estado nutricio en la población con DI es la dificultad para lograr la participación de los padres/cuidadores, como quedó claro en un estudio realizado a 15 niños con SD, donde fue complicado el involucramiento de sus cuidadores. ${ }^{3}$ En otro estudio se encontró que las mamás de niños con parálisis cerebral presentan problemas con las prácticas de alimentación de sus hijos, ya que no existe variedad en la dieta y no se incluye al niño en la dieta familiar. ${ }^{9}$

De acuerdo al Censo de Olimpiadas Especiales, las causas de discapacidad intelectual son: síndrome de Down, parálisis cerebral, autismo, síndromes de Noonan y Williams Beuren, y SDAH; en algunos casos no se especifica la causa de la discapacidad. Las Olimpiadas Especiales de Guanajuato cuentan con 296 atletas afiliados, distribuidos en 10 municipios; León es la delegación con mayor número de atletas, con un total de $117 .^{10}$

\section{METODOLOGÍA}

Se realizó un estudio descriptivo, prospectivo y transversal, donde participaron 179 atletas con DI y sus padres. La muestra fue tomada a simple disponibilidad y que cumpliera con todos los criterios de inclusión. ${ }^{5}$

Esta investigación se ajustó a las Normas Éticas Internacionales, a la Ley General de Salud en materia de investigación en seres humanos, y a la declaración de Helsinki. ${ }^{11}$

El consumo alimentario se obtuvo mediante un cuestionario semicuantitativo validado (el Sistema de evaluación de hábitos nutricionales y consumo de nutrimentos, SNUT). El cálculo del consumo de nutrimentos específicos se estimó con el programa de cómputo desarrollado por el Instituto Nacional de Salud Pública (INSP). Los componentes de la dieta analizados fueron el consumo de energía total, proteínas, hidratos de carbono y lípidos. El diagnóstico nutricio se determinó mediante parámetros antropométricos como peso, estatura, circunferencia de cintura e índice de masa corporal. El rendimiento físico fue evaluado por las pruebas del protocolo DIDEFI ${ }^{\circledR}$, que incluyen: salto horizontal, fuerza de la mano medida con un dinamómetro calibrado en kilogramos marca TAKEI T18, flexión del tronco mediante la técnica de sentado y alcanzar, así como abdominales por minuto. El estado de hidratación fue determinado por la densidad urinaria con la ayuda de un refractómetro marca Sper modelo 3005, en las mismas condiciones de temperatura y humedad. Para la evaluación del nivel de conocimientos y hábitos de hidratación, así como las prácticas alimentarias de los padres y/o cuidadores, se aplicaron un cuestionario validado sobre los conocimientos y hábitos relacionados con la hidratación y un cuestionario establecido por la Academia de Nutrición y Dietética.

El análisis estadístico fue realizado por el Programa Statgraphics Centurion XVversión 16.1. Los datos cuantitativos se expresaron como medias y desviación, mientras que los datos cualitativos se expresaron como frecuencia y proporción, y se determinó el nivel de significancia mediante $\chi^{2}$.

\section{RESULTADOS}

Se realizó un estudio descriptivo con una muestra total de 179 atletas con DI afiliados a las Olimpiadas Especiales de Guanajuato, pertenecientes a nueve municipios. Para fines de este estudio, la población fue clasificada por grupos de edad. Se encontró que 114 (63.69\%) atletas fueron hombres y $65(36.31 \%)$ mujeres, con una edad promedio de 16.76 años \pm 9 para hombres y $17.97 \pm 8$ para mujeres.

En el cuadro I se muestran los resultados por grupo de edad de la evaluación dietética obtenida a partir del SNUT: energía en kilocalorías (kcal), proteínas, lípidos e hidratos de carbono en gramos ( $\mathrm{g}$ ). El consumo más alto tanto de kilocalorías como de macronutrimentos se presenta en los hombres de entre 16 y 20 años.

La figura 1 presenta el diagnóstico nutricio por grupo de edad. Se observa que más de la mitad de la población pre-

Cuadro I. Resultado en gramos de la media del consumo de macronutrimentos por grupos de edad de acuerdo con la evaluación dietética obtenida a partir del Sistema de Evaluación de Hábitos Nutricionales y Consumo de Nutrimentos, SNUT.

\begin{tabular}{|c|c|c|c|c|c|c|c|c|c|}
\hline \multirow[t]{2}{*}{ Edad (años) } & \multirow[t]{2}{*}{$\mathrm{n}$} & \multicolumn{2}{|c|}{ Proteínas (g) } & \multicolumn{2}{|c|}{ Lípidos (g) } & \multicolumn{2}{|c|}{ Hidratos de carbono $(\mathrm{g})$} & \multicolumn{2}{|c|}{ Energía (kcal) } \\
\hline & & $\mathrm{X}$ & $\mathrm{DE}$ & $x$ & $\mathrm{DE}$ & $\mathrm{x}$ & $\mathrm{DE}$ & $x$ & $\mathrm{DE}$ \\
\hline$<11$ & 40 & 84.25 & 28.6 & 99.95 & 38.2 & 314.40 & 110.3 & 2494.14 & 833.2 \\
\hline $11-15$ & 55 & 87.14 & 27.3 & 109.34 & 38.4 & 330.49 & 123.8 & 2654.58 & 882.5 \\
\hline $16-20$ & 34 & 99.10 & 36.2 & 124.77 & 50.5 & 350.56 & 127.3 & 2921.58 & 1013.4 \\
\hline $21-25$ & 26 & 93.43 & 26.4 & 110.50 & 45.0 & 361.33 & 125.9 & 2813.53 & 922.1 \\
\hline $26-30$ & 7 & 90.40 & 17.9 & 110.86 & 28.9 & 313.82 & 120.6 & 2614.60 & 776.5 \\
\hline $31-35$ & 7 & 80.29 & 18.6 & 97.34 & 29.2 & 302.30 & 85.5 & 2406.46 & 513.2 \\
\hline$>36$ & 10 & 92.71 & 30.5 & 111.21 & 40.4 & 340.72 & 111.9 & 2734.60 & 883.0 \\
\hline Total & 179 & 89.86 & 29.1 & 110.04 & 42.3 & 334.02 & 119.3 & 2685.91 & 889.9 \\
\hline
\end{tabular}




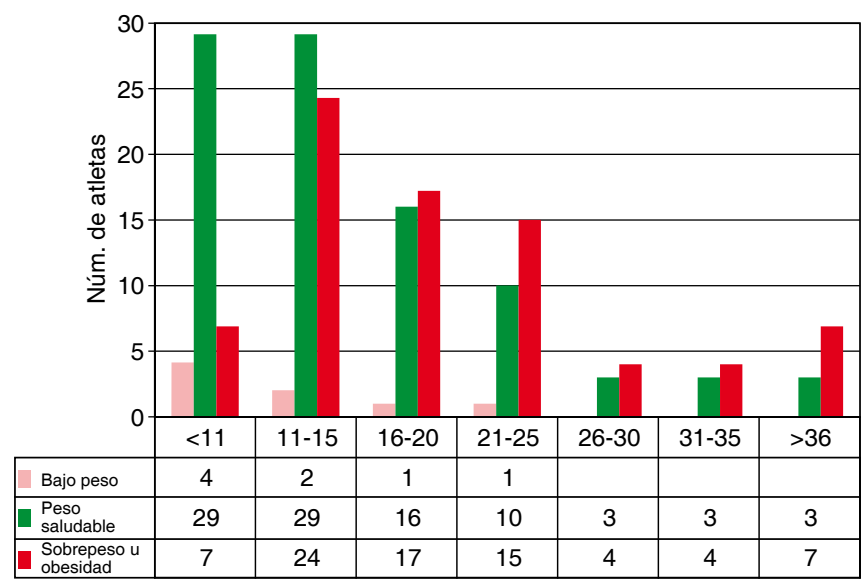

Figura 1. Diagnóstico nutricio por grupo de edad.

senta un peso saludable, aunque a partir de los 16 años el número de atletas con sobrepeso u obesidad es más alto que el de aquellos con un peso saludable. Sólo se encontraron ocho atletas $(4.47 \%)$ con bajo peso.

En lo referente a los resultados obtenidos por grupo de edad en las pruebas físicas, se encontró que la media más alta fue en el salto horizontal $(X=74.36 \pm 39)$, abdominales por minuto $(15.63 \pm 9.1)$ y fuerza de la mano por dinamometría (22.64 \pm 8.4) en el grupo de edad de 26 a 30 años; y flexión $(5.67 \pm 5.9)$ en atletas menores de 11 años.

En las figuras 2 a 5 se muestran los resultados de las medias de las pruebas físicas por grupo de edad y tipo de discapacidad. Se observó que en las pruebas de salto horizontal, fuerza de manos y abdominales por minuto, los valores más altos se presentaron en las personas con DI sin SD en todos los grupos, excepto el de 26 a 30 años de edad. La flexibilidad es mayor en las personas con SD. Además, se encontró una tendencia al crecimiento monotónico en la fuerza de las manos y abdominales por minuto, una tendencia parabólica en el salto horizontal y una representación azarosa en la flexión del tronco.

El diagnóstico cualitativo del estado de hidratación se determinó como adecuado para la población con una densidad urinaria $<1.021$. Se encontró que el $56.42 \%$ de la población en general presenta deshidratación; la media de densidad urinaria en el grupo de estudio fue de $1.022 \pm 0.01$.

En relación con los conocimientos de hidratación, el 56\% de los padres y/o cuidadores presentan un nivel de conocimientos malo o mínimo; así mismo, el 79\% de los padres presentan malos hábitos de hidratación. En la figura 6 se muestran los resultados cualitativos del cuestionario de conocimientos y hábitos de hidratación de los padres y/o cuidadores. Se encontró que hay una diferencia estadísticamente significativa entre los resultados del cuestionario

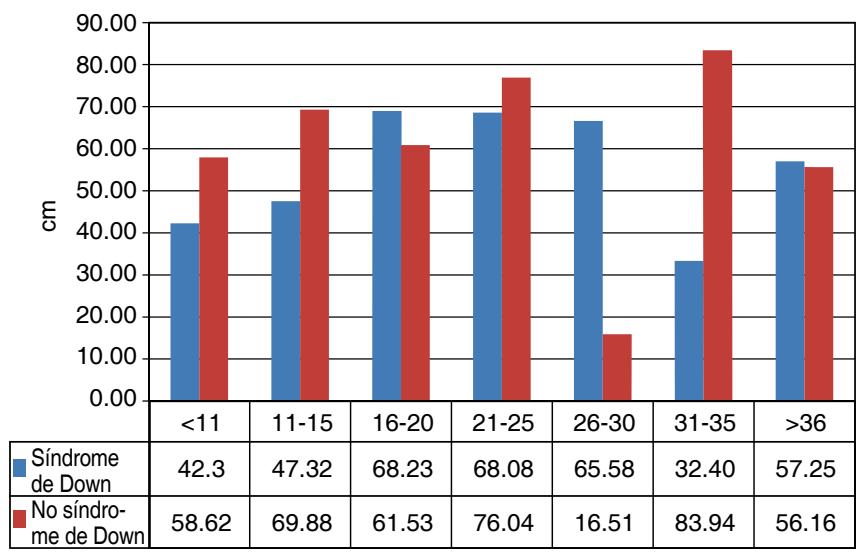

Figura 2. Resultado en centímetros de la prueba de salto horizontal por tipo de discapacidad.

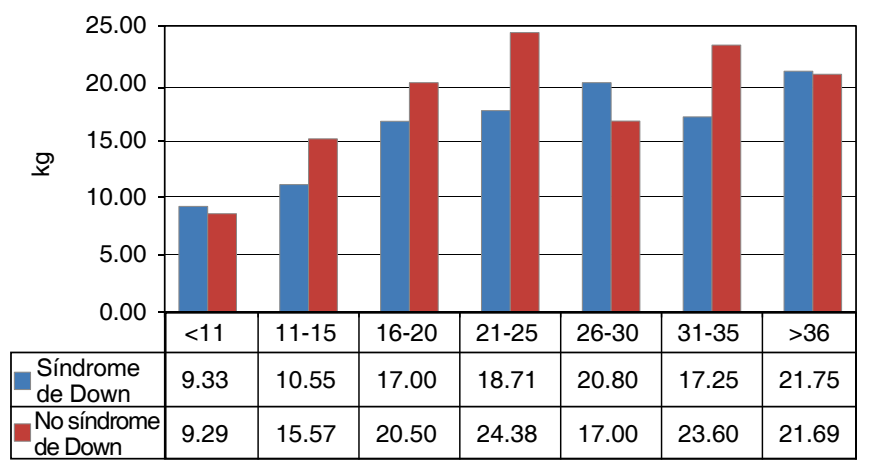

Figura 3. Resultado en kilogramos de la prueba de fuerza de mano por dinamometría por tipo de discapacidad.

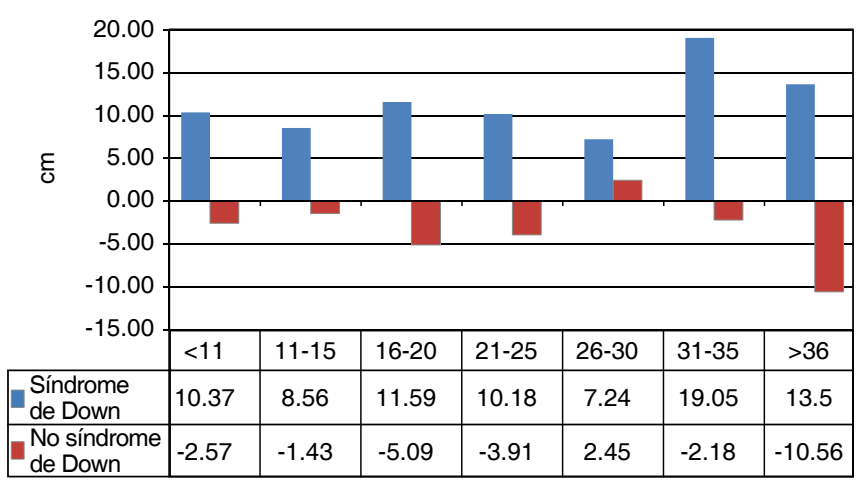

Figura 4. Resultado en centímetros de la prueba de flexión por tipo de discapacidad.

de conocimientos y del de hábitos de hidratación $(\mathrm{p}=$ 2,54037E-16).

Con respecto al cuestionario de "hábitos saludables" contestados por los padres, el $57 \%$ obtuvo un puntaje entre 13 y 16, lo que significa que pertenece a una familia que está en general bien encaminada, pero podrían esforzarse para cambiar aquellas áreas en las 


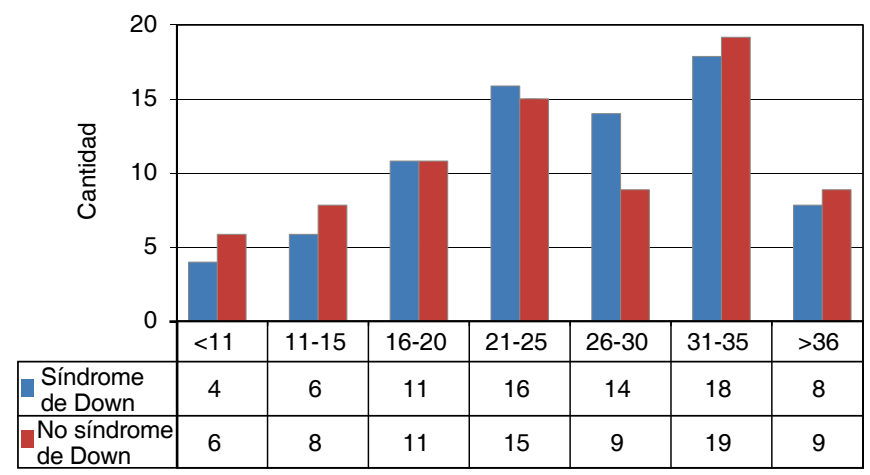

Figura 5. Resultado de la prueba de abdominales por minuto por tipo de discapacidad.

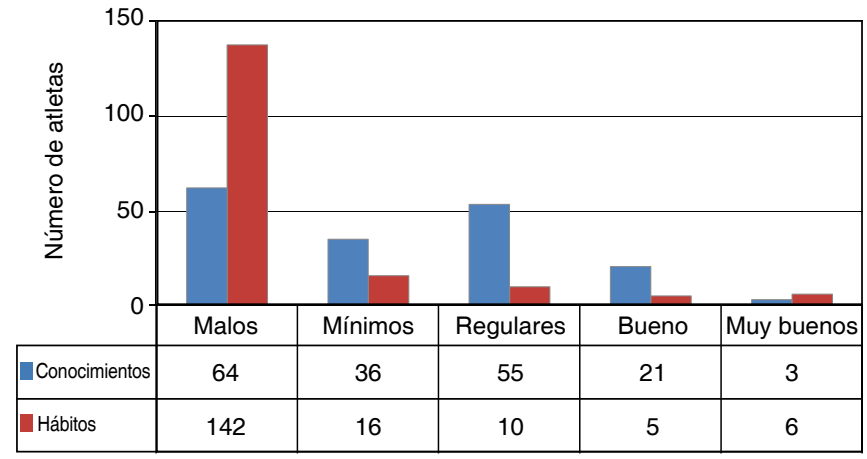

$\chi 2$ con un nivel de significancia $\mathrm{p}=2,54037 \mathrm{E}-16$.

Figura 6. Resultados de la calificación cualitativa del cuestionario sobre conocimientos y el de hábitos de hidratación en padres y/o cuidadores.

que respondieron "no" o "a veces." El $40 \%$ obtuvo un puntaje de 20-22 que indica que la familia va por buen camino, por lo que se recomienda la utilización de la guía recomendada por la Academia de Nutrición y Dietética para obtener ideas adicionales sobre hábitos sanos de alimentación y actividad física.

\section{DISCUSIÓN}

En la evaluación dietética, se encontró un consumo mayor al porcentaje de adecuación de calorías y lípidos, al igual que lo reportado por Fernández, con ciertas diferencias: en nuestro estudio se observó un consumo menor de hidratos de carbono y proteínas, y en el suyo, la ingesta fue alta en hidratos de carbono y normal en proteínas. ${ }^{5}$

En el trabajo de González se encontró que el $73.6 \%$ de la población adolescente con SD presenta sobrepeso u obesidad, cifras mayores a del presente estudio. Soler reportó un porcentaje más alto en comparación con los sujetos de este estudio, con $73.6 \%$ de adultos jóvenes que presentan sobrepeso u obesidad. ${ }^{13,14}$
En la investigación de Fernández, realizada a la población con DI tipo SD, las medias de las pruebas físicas fueron: para salto horizontal sin carrera $62.80 \mathrm{~cm}$, presión de mano por dinamometría $14.69 \mathrm{~kg}$, flexibilidad del tronco 34.20 $\mathrm{cm}$, y abdominales por minuto 8.8. En tres de las pruebas existen diferencias con respecto a nuestros hallazgos: sus resultados para fuerza de la mano y abdominales por minuto son menores y la flexibilidad del tronco, mayor. Cabeza encontró en la población con SD comprendida entre los 18 y 38 años una media de fuerza de mano de 23.85 , mayor a la del presente trabajo. ${ }^{5,15}$

De acuerdo a lo descrito por George y colaboradores, otras tablas para evaluar las aptitudes físicas calificarían con un desempeño muy por debajo de lo normal en las pruebas de salto horizontal a casi el $96 \%$ de los sujetos de este estudio, y en abdominales por minuto al $99 \%$. En esas tablas, $137 \mathrm{~cm}$ para el salto de longitud sin carrera y 34 abdominales por minuto son los valores mínimos estimados. Por otra parte, en cuanto a las pruebas de flexibilidad y dinamometría, los valores que presentaron los sujetos de estudio de acuerdo a la batería de pruebas físicas Eurofit podrían clasificarse dentro de los criterios recomendados, pero su uso no está validado para las personas con DI. ${ }^{15,16}$

En una investigación realizada a deportistas infantiles y juveniles sin DI, se encontró que el $62.22 \%$ presentaba alteraciones en el estado de hidratación, lo cual difiere a lo reportado en el presente estudio, $56.42 \% .^{12}$

En lo que concierne a los conocimientos y hábitos de hidratación, cualitativamente se encontró que hay una diferencia estadísticamente significativa entre los resultados del cuestionario de conocimientos y los del de hábitos de hidratación. Esto coincide con Escalante, quien halló en un estudio realizado a deportistas niños y adolescentes sin DI que, aunque en conocimientos sobre hidratación se obtienen mejores calificaciones, el porcentaje de personas con adecuados hábitos de hidratación es menor. ${ }^{12}$

Sobre los hábitos saludables, en el presente trabajo se observa que el porcentaje más alto es de un $57 \%$, que presentó un puntaje de 13 a 16; el 3\% presentó un puntaje menor a 12, lo cual difiere de lo reportado por Adolfsson y Draheim: ambos reportan que las personas con discapacidad intelectual presentan pobres hábitos dietéticos. ${ }^{17}$

Finalmente, y ante el insuficiente nivel de conocimiento en temas de rendimiento físico, hidratación en deportistas y conocimientos de hidratación y nutrición en atletas con DI, es necesario continuar con esta línea de estudio, dado que el campo de investigación en el tema es amplio y se requiere que más profesionistas se involucren para estudios posteriores en la población mexicana. 


\section{CONCLUSIONES}

El grupo de edad de 11 a 20 años presenta los valores más altos tanto en calorías como en macronutrimentos.

Se pudieron categorizar las pruebas físicas de acuerdo al grupo de edad y sexo.

El $56.42 \%$ de la población evaluada presenta deshidratación, sin existir diferencias estadísticamente significativas por el tipo de discapacidad.

El 56\% de los cuidadores encuestados muestra malos y mínimos conocimientos sobre hidratación en deportistas, y el 79\% obtuvo la misma calificación en hábitos de hidratación aplicados a los atletas; además existe una diferencia estadísticamente significativa en los resultados entre ambos cuestionarios. En cuanto a la evaluación para la aplicación de buenos hábitos familiares, solo el 3\% obtuvo menos de 12 de 22 como puntaje total.

Finalmente es importante resaltar que los atletas con discapacidad intelectual deben considerarse como un grupo de riesgo por presentar factores como alteraciones en el peso, además de que, debido a su condición, muchos de ellos dependen de su cuidador principal, cuya la falta de conocimientos en nutrición e hidratación puede poner en riesgo la salud y desempeño deportivo del atleta.

\section{AGRADECIMIENTOS}

Gracias a Fondos Mixtos de CONACYT-CONCYTEG por su apoyo financiero. Proyecto No. 166400.

\section{BIBLIOGRAFÍA}

1. Organización Mundial de la Salud. Discapacidades [Internet]. [Consultado el 30/02/12]. Disponible en: http://www.who.int/topics/disabilities/es/

2. Instituto Nacional de Estadística y Geografía. Porcentaje de la población con discapacidad según tipo de discapacidad para cada entidad federativa [Internet]. 2000. [Consultado el 21/02/12]. Disponible en: http://www. inegi.gob.mx
3. Le Roy C, Rebollo RJ, Moraga F, Díaz J, Castillo C. Nutrición del niño con enfermedades neurológicas prevalentes. Rev Chil Pediatr. 2010; 81 (2): 103-113.

4. Zurita F, Martínez R, Ali OJ, Fernández R, Fernández M. Aportaciones a la determinación de la prevalencia de la obesidad entre el alumnado de educación especial. Rev Pediatr Aten Primaria. 2010; 12 (45): $15-31$.

5. Fernández MC. Relación entre el consumo alimentario y rendimiento físico en atletas con síndrome de Down [Tesis de Licenciatura]. León, Guanajuato, México: Universidad Iberoamericana León; 2008. pp. 18, 25-28, 35-37.

6. Bofill AM. Educación física en personas con DI: una propuesta para evaluar manifestaciones de la condición física de manera inclusiva. Edu y Diver. 2010; 4 (2): 17-32.

7. Rivera AE, Sánchez JM, Escalante J, Caballero O. Utilidad de la densidad urinaria en la evaluación del rendimiento físico. Rev Mex Patol Clin. 2008; 44 (5): 239-253.

8. Sánchez G. Tratamiento nutricio a escolares obesos y orientación alimentaria a los padres y a estos para mejorar su estado nutricio [Tesis de Licenciatura]. León, Guanajuato, México: Universidad Iberoamericana León; 2008. p. 33.

9. Montes de Oca L. Identificación de conocimientos sobre hábitos alimentarios en niños con parálisis cerebral infantil del centro de rehabilitación infantil Teletón Aguascalientes, para el diseño de una guía de orientación alimentaria dirigida a padres de familia [Tesis de Licenciatura. León, Guanajuato, México: Universidad Iberoamericana León; 2009. p. 33.

10. Censos de registros de atletas de las oficinas de Olimpiadas especiales. [Consultado el 03/03/2012.]

11. Secretaría de Salud. Título quinto: Investigación para la Salud [Internet]. [Consultado el 30/02/12]. Disponible en: http://www.salud.gob.mx/ unidades/cdi/legis/lgs/index-t5.htm

12. Escalante J. Conocimientos, hábitos y actitudes de la reposición de líquidos y su influencia en el estado de hidratación en deportistas selectivos infantiles y juveniles en León, Gto. [Tesis de Licenciatura]. León, Guanajuato, México: Universidad Iberoamericana León; 2007. pp. 6, 9-10, 20.

13. González A, Vicente G, Moreno L, Casajús A. Dimorfismo sexual en grasa corporal en adolescentes con síndrome de Down. Rev Esp de Ob. 2010; 8 (1): 214-219.

14. Emerson E. Underweight, obesity and exercise among adults with intellectual disabilities in supported accommodation in Northern England. J Intellect Disabil Res. 2005; 49 (2): 134-143.

15. Cabeza R, Centeno R, Sánchez E et al. La fuerza de prensión manual en adultos deportistas con síndrome de Down. Influencia del género y la composición corporal. Rev Andal Med Deporte. 2009; 2 (4): 116-119.

16. George JD, Fisher AG, Vehrs PR. Testes y pruebas físicas. 4a ed. España: Editorial Padiotribo; 2002. p. 177.

17. Draheim CC, Stanish HI, Williams DP, McCubbin JA. Dietary intake of adults with mental retardation who reside in community settings. Am J Ment Retard. 2007; 112 (5): 392-400. 